\title{
Graph Based Cooperative Localization Using Symmetric Measurement Equations and Dedicated Short Range Communication*
}

\author{
Dhiraj Gulati $^{1,4}$, Feihu Zhang ${ }^{2}$, Daniel Clarke ${ }^{3}$, and Alois Knoll ${ }^{4}$
}

\begin{abstract}
Safety is one of the critical challenges for a semi or fully automated driving assistance systems. One of the key parameters for a safe automated driving assistance system is precise localization of the self and surrounding vehicles. Our previous work demonstrated the use of Symmetric Measurement Equations (SME) in a Factor Graph framework and exploited the use of sensor located outside the vehicle. In this paper we present a new approach which not only performs above mentioned cooperative vehicle infrastructure localization but also uses Dedicated Short Range Communication (DSRC). This work goes further in the direction of a complete V2X solution not only involving the infrastructure sensor but also the neighbouring vehicles. DSRC has been increasingly incorporated in all the the modern vehicles. Better state estimation is achieved by formulating the range information from DSRC as a new DSRC Range Factor in the Factor Graph. Simulations indicate better performance over the previously proposed approach of only using SME in the Factor Graph, thereby progressing a step further towards safe automated driving assistance systems.
\end{abstract}

\section{INTRODUCTION}

Semi or fully automated driving assistance system is heavily dependent on subsystems like collision avoidance and path planning for safety. These subsystems further expect a precise localization of ego and neighboring vehicles in a common coordinate framework. With decreasing hardware costs, amount of sensors available inside and outside the vehicle have increased. And along with the possibility of sharing data in real time has revolutionized the Vehicle to Vehicle (V2V) and Vehicle to Infrastructure (V2I) technologies. Hence, the task of Cooperative Localization (CL) has become a feasible solution for safe autonomous vehicles [1].

Cooperative Localization is an old concept. [2] and [3] use Kalman Filter and its derivatives to perform the CL. Other researches provided novel solutions including Maximum A Posteriori Estimation (MAP) [4], Particle Filters [5], Markov localization [6], Split Covariance Intersection Filter [7], and Random Finite Set framework (RFS) [8].

Howard et. el. [9] uses Maximum Likelihood Estimation (MLE) to achieve the CL by combining relative measure-

\footnotetext{
*This work was supported by fortiss http://www. fortiss.org/ and BMWi IKT III SADA Project http://www.projekt-sada.de/.

${ }^{1}$ Dhiraj Gulati is with the fortiss $\mathrm{GmbH}$, München, gulatidfortiss.org

${ }^{2}$ Feihu Zhang is with the School of Marine Science and Technology, Northwestern Polytechnical University, 710072, Xi'an, China; feihu. zhangenwpu. edu. cn

${ }^{3}$ Daniel Clarke is with Cogsense Technologies Limited, Berkshire, RG14 1QL England, UK; daniel.clarke@cogsense.co.uk

${ }^{4}$ Dhiraj Gulati and Alois Knoll are with Technische Universität München, Garching bei München, Germany, dhiraj.gulati@tum.de, knollein.tum.de
}

ments between robots in a least square formulation and solving the same. Ahmad et. el. [10] does the same but also includes moving landmarks. Gulati et. el. [11] formulates the CL as a graphical model, adding sensor measurements as SMEs to formulate the joint state of the system. This is implemented as a factor graph within the Georgia Tech Smoothing and Mapping (GTSAM) [12] framework.

Since early 2000 radio based communications have been explored to perform localizations for sensor networks[13]. And in the last decade concept has been extended to develop protocols and algorithms to localize the vehicles using Vehicular Ad-hoc Networks (VANETs) [14]. This has evolved into DSRC. Various researchers have proposed novel ways to use DSRC to perform CL. Parker et. al. [15] uses signal strength of DSRC to calculate the inter node distance. It then performs CL by fusing inter node distance, road maps and vehicle kinematics using Extended Kalman Filter. Mohammadabadi et. al. [16] uses inter-node distances determined by Positive Orthogonal Codes in combination with semiextended Kalman Filter to perform CL.

Mensing et. al. [17] use range information calculated by time of difference of arrival from 3GPP-LTE. They then use Kalman Filter and Particle Filter to fuse and perform CL. Alam [18] performs CL using DSRC and other sensor information like infrastructure nodes and GPS. He not only relies on range of vehicle but also on range-rate. He demonstrates his results using Kalman Filter, Extended Kalman Filter and Particle Filter.

Our previous work [11] using Factor Graph and SME successfully solves the problem of CL. Further it also avoids data association and scales optimally for non-linear cases. In this paper we propose the novel idea of use of a DSRC Range Constraint Factor, in addition to the SME Factor, into a factor graph to perform CL for semi or full autonomous vehicles. Our simulations indicate that the new combination of DSRC Range and SME Factor for CL performs better than the stand alone SME Factor.

Rest of the paper is organized as follows. Section II defines the problem with its constraints. Section III and IV give the overview of the SME and DSRC respectively, which is followed by explanation of Factor Graphs and Non linear Least Square Optimization methods in Section V. Section VI presents the evaluation and VII concludes the paper.

\section{PROBLEM DESCRIPTION}

A typical scenario which we would like to address by vehicle-infrastructure CL is shown in (Fig. 1(a)). Various assumptions are: 


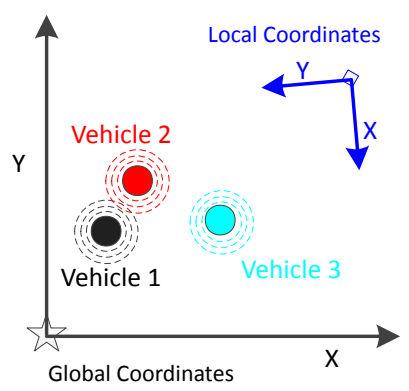

(a)

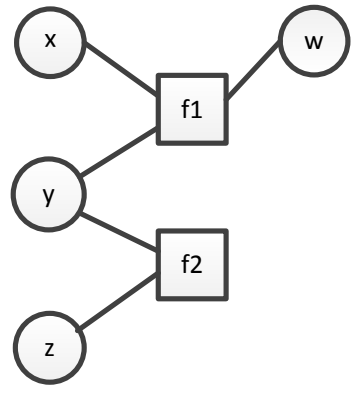

(b)
Fig. 1. (a) Three vehicles represented with solid circles. Local Coordinate System in blue is of RADAR. Dotted circles around the circles represent the DSRC communication field of the vehicle. (b) Factor graph with variables $w, x, y, z$ and functions $f 1(w, x, y)$ and $f 2(y, z)$.

- Each vehicle has an Odometry and a GPS sensor to localize itself in an absolute reference and can broadcasts its measurements.

- Each vehicle has the capability to perform ranging using DSRC. Ranging is the method to uniquely identify the vehicle and its distance from the ego vehicle.

- The infrastructure RADAR sensor can derive the global position of all the vehicles in its field of view, but cannot uniquely identify the vehicles. This introduces a challenge from the perspective of data association.

- The vehicles and RADAR Sensor can communicate in both directions without any timing delay or data error.

- The environment has no clutter and there are no miss detections.

The goal of vehicle-infrastructure CL is to improve the precision of position estimates of the participating vehicles.

\section{Symmetric Measurement EQuations}

The aim of the SME is to generate 'pseudo-measurements' using symmetric functions from the original measurements [19]. This is achieved by adding or multiplying the sensor measurements to generate new measurements having values from all the targets. The resulting measurements are independent of the targets, thereby avoiding a computationally expensive data-association step. Following are two SME representations of the three targets in 1 dimension:

- Sum-of-powers:

$$
S_{\text {pow }}=\left[\begin{array}{l}
m_{1}+m_{2}+m_{3} \\
m_{1}^{2}+m_{2}^{2}+m_{3}^{2} \\
m_{1}^{3}+m_{2}^{3}+m_{3}^{3}
\end{array}\right]
$$

- Sum-of-product:

$$
S_{\text {prod }}=\left[\begin{array}{c}
m_{1}+m_{2}+m_{3} \\
m_{1} m_{2}+m_{2} m_{3}+m_{1} m_{3} \\
m_{1} m_{2} m_{3}
\end{array}\right]
$$

where $m_{i}$ is the measurement from the $i^{t h}$ target.

For further details reader can refer to [19] and [11].

\section{DedicAted Short Range COMmunicAtion}

For real time wireless communication, a dedicated Wireless Local Area Network (WLAN) protocol IEEE802.11p has been proposed. U.S. Federal Communication Commission has already allocated $75 \mathrm{MHz}$ in the $5.9 \mathrm{GHz}$ band in 1999 for the same [20]. Similarly European Telecommunication Standards Institute (ETSI) allocated $30 \mathrm{MHz}$ in $5.9 \mathrm{GHz}$ band [21]. Various other authorities around the world have also reserved dedicated bandwidth. This bandwidth is called DSRC. DSRC is for data-only systems and has a range for about $1000 \mathrm{~m}$ for Line-Of-Sight conditions. But practically this range is lower and highly dependent on obstacles and objects in the environment. Most of the modern day cars capable of semi or fully automated driving are equipped with the DSRC transceivers and transponders.

One of the important property of DSRC is that using DSRC vehicles can be uniquely identified. Since we want to estimate the distance of neighboring uniquely identified vehicles from the ego vehicle, we only concentrate on the methodologies using DSRC to find the same. There are various radio-ranging methodologies like received signal strength (RSS), time of arrival (TOA), time difference of arrival (TDOA), Doppler Shift and Angle of Arrival (AOA). Each of the methods have their benefits and problems.

Like the name suggests RSS uses the received strength/power of the signal to estimate the range. The attenuation of signal strength is governed by the path loss model and this is used for range estimation. TOA/TDOA use signal propagation time to estimate the distance and requires the clocks of sender and reviver to be synchronized. Doppler Shift is the difference between emitted frequency and observed frequency. Using the combination of vehicular speeds from the data over DSRC and doppler shift the range can be calculated. AOA is used to get the bearing of received signals.Interested reader can refer Alam et. al. [22] for details of the above methods along with the performances of each of them.

\section{NONLINEAR LEASt SQUARE Optimization}

\section{A. Factor Graphs}

A factor graph is a bipartite graph $G_{k}=\left(F_{k}, V_{k}, E_{k}\right)$ with two types of nodes: factor nodes $f_{i} \in F_{k}$ and variable nodes $v_{j} \in V_{k}$. Edges $e_{i j} \in E_{k}$ can exist only between factor nodes and variable nodes, and are present if and only if the factor $f_{i}$ involves a variable $v_{j}$ [23]. It can also represent Probabilistic Graphical Models (PGM) and used to implement Bayesian networks [24] and Markov random fields [25].

Fig. 1(b) is an example of a factor graph with variables $w, x, y, z$ and functions $f 1$ and $f 2$ with factorization: $f(w, x, y, z)=f 1(w, x, y) * f 2(y, z)$. Using PGM, the example can also be represented as $P(w, x, y, z)=$ $P(w, x, y) * P(y, z)$. Similarly we use the factorized probability distribution to represent the entire trajectories of all the participating vehicles as an optimization problem. The localization can then be represented as by estimating the trajectory $\mathbf{x}=\left\{x_{i} \mid i \in 0, \ldots, n\right\}$, for a given set of measurements from various sensors, for example from Sensor-1 
$\mathbf{z}^{1}=\left\{\mathbf{z}^{1} \mid i \in 0, \ldots, n\right\}$, Sensor- $2 \mathbf{z}^{2}=\left\{z^{2} \mid i \in 0, \ldots, n\right\}$ and Odometry $\mathbf{u}=\{u \mid i \in 0, \ldots, n\}$. Thus the joint density for the measurements from the three Sensors can be represented as:

$$
P\left(\mathbf{x}, \mathbf{z}^{1}, \mathbf{z}^{2}, \mathbf{u}\right) \propto P\left(x_{0}\right) \prod_{i}^{n} P\left(x_{i+1} \mid x_{i}, u_{i}\right) \prod_{k}^{m} P\left(z_{k} \mid x_{i k}\right)
$$

where $z_{k} \in\left\{\mathbf{z}^{1}, \mathbf{z}^{2}\right\}$ denotes the measurement, originating from either Sensor-1 or Sensor-2.

Now the factorization is done based on Gaussian distributions for the process and measurement models as:

$$
\begin{gathered}
x_{i}=f_{i}\left(x_{i-1}, u_{i}\right)-w_{i} \Leftrightarrow P\left(x_{i+1} \mid x_{i}, u_{i}\right) \\
\propto \exp \left(-\frac{1}{2}|| f_{i}\left(x_{i-1}-x_{i} \|_{\Gamma_{i}}^{2}\right)\right. \\
z_{k}=h_{k}\left(x_{i k}\right)-v_{k} \Leftrightarrow P\left(z_{k} \mid x_{i k}\right) \\
\propto \exp \left(-\frac{1}{2}|| h_{k}\left(x_{i k}\right)-z_{k} \|_{\Sigma_{k}}^{2}\right)
\end{gathered}
$$

where $h$ and $f$ denote the measurement and process models, and $v$ and $w$ are the corresponding noises with covariance matrices $\Sigma_{k}$ and $\Gamma_{i}$.

In this paper, the goal is to calculate the maximum likelihood estimation (MLE) by using the nonlinear least square method:

$$
\begin{aligned}
& \bar{\theta}=\operatorname{argmax} P(\theta \mid \mathbf{z}, \mathbf{u})= \\
& \operatorname{argmin}\left\{\sum_{i=1}^{n}\left\|f_{i}\left(x_{i-1}-u_{i}\right)-x_{i}\right\|_{\Gamma_{i}}^{2}+\sum_{k=1}^{m}\left\|h_{k}\left(x_{i k}\right)-z_{k}\right\|_{\Sigma_{k}}^{2}\right\}
\end{aligned}
$$

Next we look at the formulations of factors.

\section{B. Odometry Factor}

For a constant velocity model, the error function of the binary factor $f^{O D O M}$ between the states $X_{t}, X_{t-1}$ :

$$
f^{O D O M}\left(X_{t}, X_{t-1}\right) \triangleq d\left(z_{t}^{o}-h^{o}\left(z_{t-1}^{o}\right)\right)
$$

where $h^{o}$ is the function to calculate the odometry measurement at time $t$ and $z_{t}^{o}$ is the measurement at time $t$

The covariances provided by the sensor manufacture are used while formulating the corresponding factors.

\section{GPS Factor}

The GPS measurement results in an unary factor $f^{G P S}$ for a state $X_{t}$, which is written as:

$$
f^{G P S}\left(X_{t}\right) \triangleq d\left(z_{t}^{g}-h^{g}\left(z_{t}\right)\right)
$$

where $h^{g}$ is the measurement function, providing the relation between the measurement $z_{t}^{g}$ and the position of the vehicle $z_{t}$ at time $t$.

The covariances provided by the sensor manufacture are used while formulating the corresponding factors.

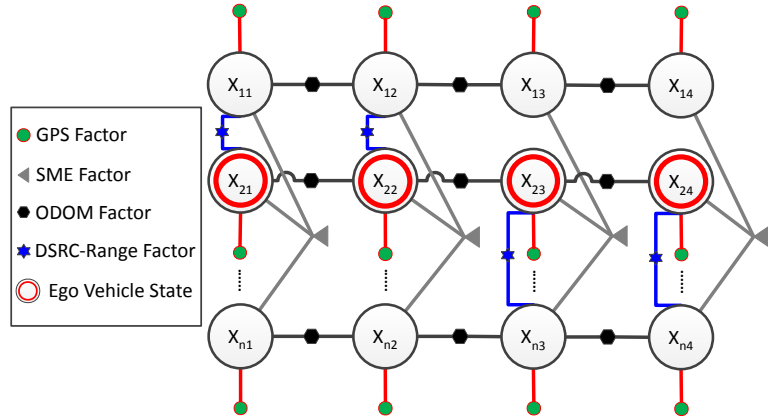

Fig. 2. Factor graph for $\mathrm{n}$ vehicles with four state nodes each and corresponding Odometry Factors, GPS Factors, SME Factors and DSRCRange Factors.

\section{SME Factor}

To perform CL, the node running the fusion also receives absolute positions, in global coordinates, of all vehicles in the field of view of RADAR. As the RADAR does not perform any data association, it will not associate the calculated positions to the individual vehicles. To incorporate such information in the factor graph, we construct the $\mathrm{N}$-ary SME Factor for states $\left(X_{0}, \cdots, X_{N}\right)_{t}$ at time $t$ as follows:

$$
f^{S M E}\left(\left(X_{0}, \cdots, X_{N}\right)_{t}\right) \triangleq d\left(z_{t}^{s}-h^{s}\left(z_{0}, \cdots, z_{N}\right)\right)
$$

If $\sigma_{x}^{2}$ and $\sigma_{y}^{2}$ are the $X$ and $Y$ variances respectively for the RADAR, then we have (see [26] for more details) $\operatorname{Cov}(x, y)=\left[\sigma_{x_{1}}^{2}, \cdots, \sigma_{x_{n}}^{2}, \sigma_{y_{1}}^{2}, \cdots, \sigma_{y_{n}}^{2}\right]$. Then we obtain the covariance for the topology estimate at any time $t$ as:

$$
\sigma_{t o p_{x, y}}^{2}=M \cdot \operatorname{Cov}(x, y) \cdot M^{T}
$$

where $M$ is a $1 X 2 N$ matrix as follows:

$$
M=\left[\frac{\partial}{\partial x_{1}}\left(z_{t}^{s}\right), \cdots, \frac{\partial}{\partial x_{n}}\left(z_{t}^{s}\right), \frac{\partial}{\partial y_{1}}\left(z_{t}^{s}\right), \cdots, \frac{\partial}{\partial y_{n}}\left(z_{t}^{s}\right)\right]
$$

\section{E. DSRC Range Factor-the novel idea}

For an ego vehicle, the distance between any neighboring vehicle $v$ in the DSRC range can be calculated as:

$$
z_{t}^{d s r c}=h^{d s r c}\left(v_{t}\right)+n^{d}
$$

where $h^{d s r c}$ is any of the range calculating functions as described in the Sec. IV at time $t . n^{d}$ represents the measurement noise. Eq. (12) gives a binary factor between the states of ego $\left(X_{t}^{e}\right)$ and neighboring $\left(X_{t}^{v}\right)$ vehicles as:

$$
f^{d R a n g e}\left(X_{t}^{e}, X_{t}^{v}\right) \triangleq d\left(z_{t}^{d s r c}-h^{d s r c}\left(v_{t}\right)\right)
$$

As the distance can be calculated by any of the methods based on DSRC as mentioned in Sec. IV. Hence the error in measurement depends on the method used and is adjusted while formulating the corresponding factors.

Fig. 2 shows the factor graph with four kinds of factors.

\section{F. Smoothing}

The formulated factor graph is solved using the Levenberg Marquardt Optimizer. Using an initial estimate $x_{0}$ it iteratively finds an update $\Delta$ from the linearized system:

$$
\underset{\Delta}{\arg \min } J\left(x_{0}\right) \Delta-b\left(x_{0}\right)
$$




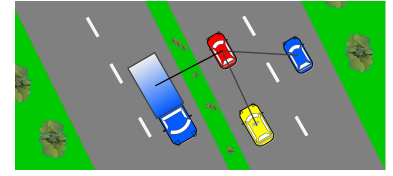

(a)

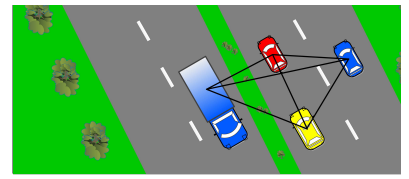

(b)
Fig. 3. (a) Star Architecture. (b) Cluster Architecture.

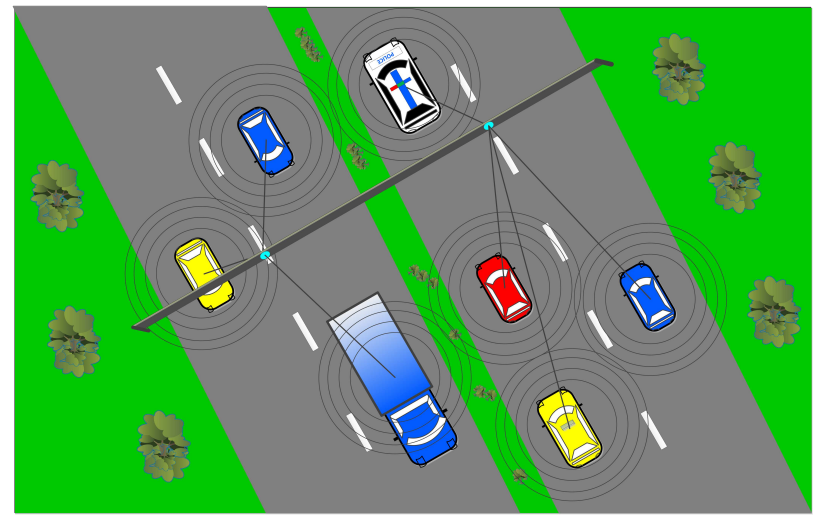

Fig. 4. Simulation scenario of a highway with vehicles. Simulated RADAR is mounted above the ground on a beam across the highway. The RADAR is assumed to have a filed of view in both the directions. The vehicles represent the approximate state of their trajectory at some time $t$. The circles around the vehicles represent the DSRC signal.

where $J\left(x_{0}\right)$ is the sparse Jacobian Matrix at the current linearization point $x_{0}$ and $b\left(x_{0}\right)=f\left(x_{0}\right)-z$ is the residual for given the measurement $\mathrm{z}$. After solving (14), the linearization point is updated to the new estimate $x_{0}+\Delta$. Further detail on this process is presented within [27].

The Jacobian for Odometry and GPS is $\operatorname{diag}[1,1]$ and for DSRC Range is 1 . The Jacobian for each of $p^{\text {th }}$ SME Measurement equation $(p=1 \cdots n)$ is:

$$
M=\operatorname{diag}\left[\frac{\partial\left(\sum_{i=1}^{n}\left(x_{i}^{p}\right)\right)}{\partial x}, \frac{\partial\left(\sum_{i=1}^{n}\left(y_{i}^{p}\right)\right)}{\partial y}\right] \forall x, y
$$

\section{Evaluation}

\section{A. Simulation Setup}

CL using DRSC can be primarily done using Star Architecture or Cluster Architecture (fig (3)). Since we only concentrate on direct distance measurement using DSRC we only consider the scenario of star architecture. The other needs more information to be sent from the participating vehicles.

We simulate a highway scenario with 16 vehicles with an infrastructure RADAR mounted across the lanes of the highway (Fig. (4)). We assume the infrastructure RADAR has equal field of view in both the directions. We simulate the various tests for 200 time steps. GTSAM [12] is used to implement the factor graphs.

Simulated vehicles provide their own odometry measurements and location in global coordinates. Infrastructure RADAR provides global coordinates for vehicles in its field of view without performing any data association. The noises are assumed as $\operatorname{diag}[1.0,1.0], \operatorname{diag}[10.0,10.0]$ and $\operatorname{diag}[0.5,0.5]$ for the Odometry, the GPS and the RADAR respectively.
Alan et. al. [22] point the error for various ranging methodologies using DSRC from as good as $1 \mathrm{~m}$ and worse even upto $10 \mathrm{~m}$. So instead of using one error value we run our simulations for error in range measurement between $1 m-6 m$. Also as mentioned DSRC signals have the range from $100 m$ to $1000 m$, with $1000 m$ difficult to achieve. For the simulations of the ego vehicle (depicted in red in fig ((4)) we use DSRC range from $100 \mathrm{~m}$ to $600 \mathrm{~m}$.

Results from the simulation are compared three ways, between:

- the fused trajectory only using Odometry and GPS;

- the fused trajectory for Odometry, GPS and SME Factor [11]; and

- the fused trajectory for Odometry, GPS, SME Factor and DSRC Range Factor.

Then the above experiments are repeated with different error in measurement and different DSRC range.

To clearly analyse the benefits of adding DSRC factors we run use MonteCarlo simulation methodology of 1000 iterations. To avoid the influence of any other Sensor measurement, we keep the simulated measurements from Odometry, GPS and RADAR same in all the 1000 iterations.

The performance is measured by calculating Root Mean Square Deviation (RMSE) value for the complete system. The total error is the sum of the RMSE of each vehicle for $n$ steps:

Error $=\sqrt{\frac{\sum_{j=1}^{n} \sum_{i=1}^{2}\left[\left(x_{i_{e s t}}-x_{i_{\text {real }}}\right)^{2}+\left(y_{i_{\text {est }}}-y_{i_{\text {real }}}\right)^{2}\right]^{j}}{n}}$

\section{B. Results}

Fig. 5 to Fig. 7 show the total system RMSE results for the simulation of the DSRC range of $100 \mathrm{~m}, 300 \mathrm{~m}$, and $600 \mathrm{~m}$. For each DSRC range we plot only the error of $1 \mathrm{~m}$ and $6 \mathrm{~m}$ against the case of no factor and SME Factor. From each of the figures it is clearly visible that the combination of SME and DSRC Factors gives a superior performance than the other two. Although the SME incorporates full information but adding DSRC Range Factor adds more information to the given factor graph and hence achieves better performance.

We only plot the RMSE for the $1 \mathrm{~m}$ and $6 \mathrm{~m}$ for all the DSRC range because the graphs for various standard deviations are very close and is difficult to notice any significant gain. This can be clearly seen seen in the table I. The table shows the final RMSE values obtained from Monte Carlo simulation of 1000 iterations The simulation was run for DSRC range for $100 m-600 m$ for the error between $1 m-6 m$. Also mentioned in the last column are the average number of DSRC Range Factors added for the various DSRC ranges (calulated in Table II).

It can be seen from the table (I) that:

- For each range, with increase in the error, generally the RMSE value increases. This is expected and implies that the lower is the error in measurement the better will be the estimation.

- For each error in the measurement, with increase in the range, generally the RMSE value decreases. This 
TABLE I

AVERAGE FINAL SYSTEM RMSE VALUES AFTER 200 STEPS FOR DIFFERENT DSRC RANGES AND DIFFERENT ERRORS FOR 1000 ITERATIONS.

\begin{tabular}{cccccccc}
\hline DSRC & \multicolumn{6}{c}{ Standard Deviation (m) } & \multicolumn{2}{c}{$\begin{array}{c}\text { Average Number of } \\
\text { Range }(\mathbf{m})\end{array}$} & $\mathbf{1}$ & $\mathbf{2}$ & $\mathbf{3}$ & $\mathbf{4}$ & $\mathbf{5}$ & $\mathbf{6}$ & DSRC Range Factors for this range \\
\hline 100 & 11.1620 & 11.1944 & 11.2368 & 11.2810 & 11.3283 & 11.3571 & 515 \\
200 & 11.0086 & 11.0841 & 11.1594 & 11.2210 & 11.2818 & 11.3270 & 633 \\
300 & 10.8503 & 10.9263 & 11.0065 & 11.0840 & 11.1600 & 11.2199 & 913 \\
400 & 10.7180 & 10.8004 & 10.8915 & 10.9846 & 11.0678 & 11.1436 & 1052 \\
500 & 10.5989 & 10.7162 & 10.8202 & 10.9219 & 11.0147 & 11.0954 & 1166 \\
600 & 10.4531 & 10.5961 & 10.7208 & 10.8378 & 10.9368 & 11.0285 & \\
\hline
\end{tabular}

is also expected as increase in the range for the same error in measurement implies more DSRC range factors can be added thereby adding more information to the factor graph and reaching a better estimation. This is confirmed by the last column of the table which shows the average number of DSRC Range Factors added to the graph for the given range.

The table (I) also presents some more interesting results:

- It can be seen the final system RMSE value for DSRC range of $100 \mathrm{~m}$ with error of $1 \mathrm{~m}$ is 11.1620 and for DSRC range of $600 \mathrm{~m}$ with error of $6 \mathrm{~m}$ is 11.0285 . The error reduces by almost $1.2 \%$. The general trend of the reducing error can be observed in the full table. Hence it can be seen that for a little more error with bigger range can perform better than the small error with smaller range. This happens because as the number of factors added to the system increase and hence also the information to the graph. This helps in reducing the overall error in the system. But this observation is true for linear increase in the error and can't be generalized because if errors increase non-linearly there may not be a significant gain.

- Lowering the DSRC range implies lowering the number of detected vehicles around the ego vehicle. This also means that the lower number of the vehicles in the whole system does not decrease the error significantly. But the total error is still upper bounded by the error of SME Factor which would be the case that no vehicle is present in the DSRC Range of the ego vehicle.

In our simulation experiments original measurements from the RADAR have been used, hence data association is avoided. Also the original measurements are used to construct the new SME measurements, therefore no information is lost during transformation. Using Factor Graphs helps in addressing the non-linear systems efficiently. With the increase in the DSRC range, the number of DSRC Range Factors added to the system can increase non-linearly. But because of the use of factor graphs this does not pose a challenge and the scalability of the proposed solution remains intact. Instead of various states and covariances with each time step, only measurements are sent, keeping the bandwidth requirements to minimum.

\section{Remarks}

Although we have successfully avoided the data association issue in the RADAR, the current results assume clutter

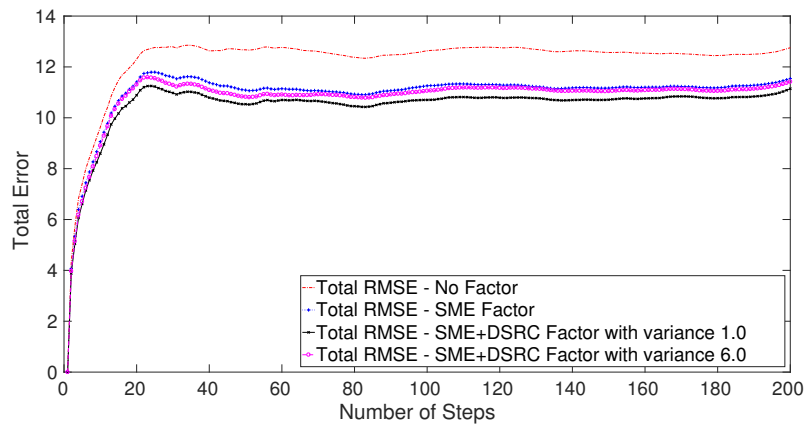

Fig. 5. Total system RMSE with DSRC range as $100 \mathrm{~m}$.

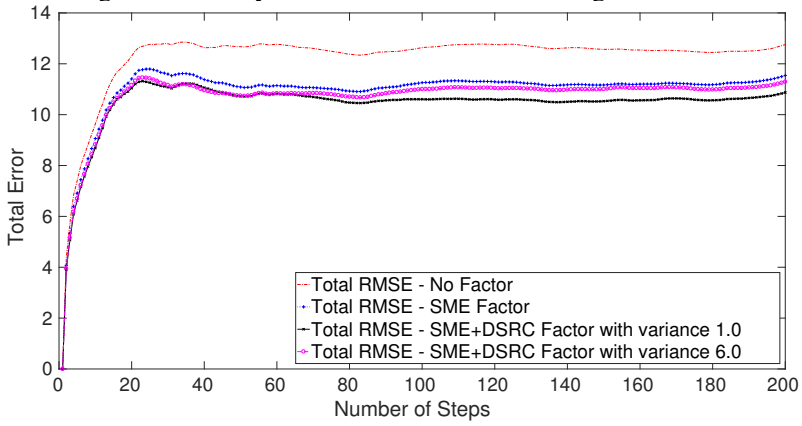

Fig. 6. Total system RMSE with DSRC range as $300 \mathrm{~m}$.

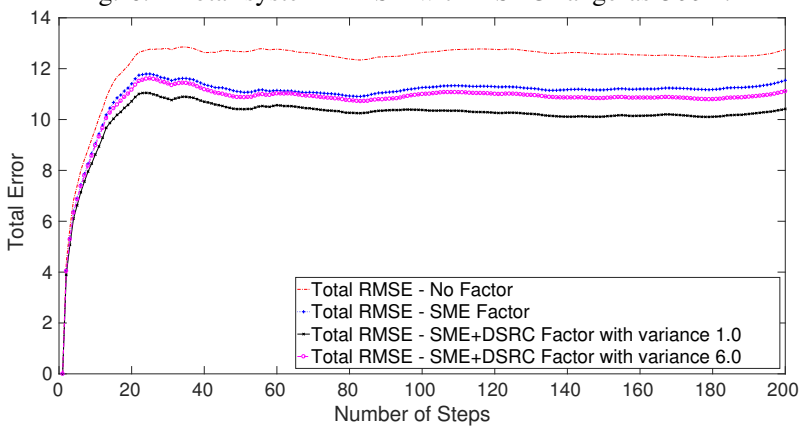

Fig. 7. Total system RMSE with DSRC range as $600 \mathrm{~m}$.

free environment. In practice this is not the case.

\section{CONCLUSION}

A novel way of improved state estimation using Dedicated Short Range Radio (DSRC) and Factor Graphs is presented in this paper. In addition to the DSRC we use RADAR measurements from the infrastructure as SME factors. The idea presented in this paper goes is the direction of achieving a complete V2X Communication mechanisms. With the presented technique we increase the precision of localization of the ego and the surrounding vehicles. Precise localization 
TABLE II

AVERAGE NUMBER OF DSRC RANGE FACTORS USED FOR 1000 ITERATIONS.

\begin{tabular}{cccccccc}
\hline & \multicolumn{7}{c}{ Standard Deviation $(\mathbf{m})$} \\
\cline { 2 - 7 } DSRC Range (m) & $\mathbf{1}$ & $\mathbf{2}$ & $\mathbf{3}$ & $\mathbf{4}$ & $\mathbf{5}$ & $\mathbf{6}$ & Average for this Range \\
\hline 100 & 515.495 & 515.362 & 515.190 & 515.214 & 515.135 & 515.342 & 515 \\
200 & 633.387 & 633.824 & 633.939 & 633.900 & 633.800 & 633.724 & 633 \\
300 & 762.224 & 762.539 & 762.578 & 762.630 & 762.626 & 762.610 & 761 \\
400 & 912.082 & 912.577 & 912.909 & 913.055 & 913.055 & 912.964 & 912 \\
500 & 1051.732 & 1051.600 & 1051.600 & 1051.761 & 1051.761 & 1051.998 & 1052 \\
600 & 1166.163 & 1166.180 & 1166.391 & 1166.615 & 1166.535 & 1166.498 & 1166 \\
\hline
\end{tabular}

increases the effectiveness of various technologies (like collision avoidance and path planning) used in semi and fully autonomous vehicles. This addresses our initial goal of safe automated driving assistance systems. By using SME we also address one of the critical problems of data association in CL. Other challenges of bandwidth issue and scalability are also addressed. In our simulation we also demonstrate the trade off of higher range vs higher error rate in the range measurement using DSRC.

Further research work is underway, considering the influence of clutter and miss-detections present in real-world systems. It will also focus on implementation with incremental smoothing for live scenarios incorporating other models like constant turn and constant acceleration. Also we investigate the capabilities of DSRC to exploit the true V2V communication, in order to get even better state estimates of the ego vehicle using Factor Graphs, using the data of communicated from in-range vehicles, about the vehicles which are not in direct range of the ego vehicle.

\section{REFERENCES}

[1] R. Kurazume, S. Nagata, and S. Hirose, "Cooperative positioning with multiple robots," in Robotics and Automation, 1994. Proceedings., 1994 IEEE International Conference on, May 1994, pp. 1250-1257 vol.2.

[2] S. I. Roumeliotis and G. A. Bekey, "Distributed multirobot localization," IEEE Transactions on Robotics and Automation, vol. 18, no. 5, pp. 781-795, Oct 2002.

[3] N. Karam, F. Chausse, R. Aufrere, and R. Chapuis, "Cooperative multi-vehicle localization," in 2006 IEEE Intelligent Vehicles Symposium. IEEE, 2006, pp. 564-570.

[4] E. D. Nerurkar, S. I. Roumeliotis, and A. Martinelli, "Distributed maximum a posteriori estimation for multi-robot cooperative localization," in Robotics and Automation, 2009. ICRA '09. IEEE International Conference on, May 2009, pp. 1402-1409.

[5] I. M. Rekleitis, G. Dudek, and E. E. Milios, "Multi-robot cooperative localization: a study of trade-offs between efficiency and accuracy," in IEEE/RSJ International Conference on Intelligent Robots and Systems, vol. 3, 2002, pp. 2690-2695 vol.3.

[6] D. Fox, W. Burgard, H. Kruppa, and S. Thrun, "A probabilistic approach to collaborative multi-robot localization," Autonomous robots, vol. 8, no. 3, pp. 325-344, 2000.

[7] H. Li and F. Nashashibi, "Cooperative multi-vehicle localization using split covariance intersection filter," IEEE Intelligent Transportation Systems Magazine, vol. 5, no. 2, pp. 33-44, Summer 2013.

[8] F. Zhang, H. Stähle, G. Chen, C. Buckl, and A. Knoll, "Multiple vehicle cooperative localization under random finite set framework," in 2013 IEEE/RSJ International Conference on Intelligent Robots and Systems, Nov 2013, pp. 1405-1411.

[9] A. Howard, M. J. Matark, and G. S. Sukhatme, "Localization for mobile robot teams using maximum likelihood estimation," in Intelligent Robots and Systems, 2002. IEEE/RSJ International Conference on, vol. 1, 2002, pp. 434-439 vol.1.
[10] A. Ahmad, G. D. Tipaldi, P. Lima, and W. Burgard, "Cooperative robot localization and target tracking based on least squares minimization," in 2013 IEEE International Conference on Robotics and Automation, May 2013, pp. 5696-5701.

[11] D. Gulati, F. Zhang, D. Clarke, and A. Knoll, "Graph-based cooperative localization using symmetric measurement equations," Sensors, vol. 17(6), no. 1422, 2017. [Online]. Available: http://www.mdpi.com/1424-8220/17/6/1422

[12] "GTSAM, Georgia Tech smoothing and Mapping," https://collab.cc.gatech.edu/borg/gtsam/.

[13] S. Gezici, Z. Tian, G. B. Giannakis, H. Kobayashi, A. F. Molisch, H. V. Poor, and Z. Sahinoglu, "Localization via ultra-wideband radios: a look at positioning aspects for future sensor networks," IEEE Signal Processing Magazine, vol. 22, no. 4, pp. 70-84, July 2005.

[14] A. Boukerche, H. A. Oliveira, E. F. Nakamura, and A. A. Loureiro, "Vehicular ad hoc networks: A new challenge for localization-based systems," Computer communications, vol. 31, no. 12, pp. 2838-2849, 2008.

[15] R. Parker and S. Valaee, "Cooperative vehicle position estimation," in ICC, 2007, pp. 5837-5842.

[16] P. H. Mohammadabadi and S. Valaee, "Cooperative node positioning in vehicular networks using inter-node distance measurements," in 2014 IEEE 25th Annual International Symposium on Personal, Indoor, and Mobile Radio Communication (PIMRC), Sept 2014, pp. 1448-1452.

[17] C. Mensing, S. Sand, and A. Dammann, "Hybrid data fusion and tracking for positioning with gnss and 3gpp-lte," International Journal of Navigation and Observation, vol. 2010, 2010.

[18] N. Alam, "Vehicular positioning enhancement using dsrc," Ph.D. dissertation, University of New South Wales, 2012.

[19] E. W. Kamen, "Multiple target tracking based on symmetric measurement equations," IEEE Transactions on Automatic Control, vol. 37, no. 3, pp. 371-374, Mar 1992.

[20] "FCC DSRC (on 31 Jan 2017)," https://www.fcc.gov/dedicated-shortrange-communications-dsrc-service.

[21] "ETSI DSRC (on 31 Jan 2017)," http://www.etsi.org/technologiesclusters/technologies/intelligent-transport/dsrc.

[22] N. Alam and A. G. Dempster, "Cooperative positioning for vehicular networks: Facts and future," IEEE Transactions on Intelligent Transportation Systems, vol. 14, no. 4, pp. 1708-1717, Dec 2013.

[23] F. R. Kschischang, B. J. Frey, and H. A. Loeliger, "Factor graphs and the sum-product algorithm," IEEE Transactions on Information Theory, vol. 47, no. 2, pp. 498-519, Feb 2001.

[24] H.-A. Loeliger, "An introduction to factor graphs," Signal Processing Magazine, IEEE, vol. 21, no. 1, pp. 28-41, 2004.

[25] V. Indelman, S. Williams, M. Kaess, and F. Dellaert, "Information fusion in navigation systems via factor graph based incremental smoothing," Robotics and Autonomous Systems, vol. 61, no. 8, pp. 721-738, 2013.

[26] K. O. Arras, "An introduction to error propagation: Derivation, meaning and examples of equation cy $=\mathrm{fx}$ cxfx t." ETH-Zürich, Tech. Rep., 1998.

[27] F. Dellaert and M. Kaess, "Square root sam: Simultaneous localization and mapping via square root information smoothing," Int. J. Rob. Res., vol. 25, no. 12, pp. 1181-1203, Dec 2006. [Online]. Available: http://dx.doi.org/10.1177/0278364906072768 